\title{
On the Use of Predictive Models for Improving the Quality of Industrial Maintenance: an Analytical Literature Review of Maintenance Strategies
}

\author{
Oana Merkt \\ Hohenheim University \\ Chair of Business Informatics II (530D) \\ Schwerzstrasse 35 \\ Stuttgart 70599, Germany \\ Email: Oana.Merkt@uni-hohenheim.de
}

\begin{abstract}
Due to advances in machine learning techniques and sensor technology, the data driven perspective is nowadays the preferred approach for improving the quality of maintenance for machines and processes in industrial environments. Our study reviews existing maintenance works by highlighting the main challenges and benefits and consequently, it shares recommendations and good practices for the appropriate usage of data analysis tools and techniques. Moreover, we argue that in any industrial setup, the quality of maintenance improves when the applied data driven techniques and technologies: (i) have economical justifications; and (ii) take into consideration the conformity with the industry standards. In order to classify the existing maintenance strategies, we explore the entire data driven model development life cycle: data acquisition and analysis, model development and model evaluation. Based on the surveyed literature we introduce taxonomies that cover relevant predictive models and their corresponding data driven maintenance techniques.
\end{abstract}

\section{INTRODUCTION}

$\mathbf{T}$ HE quality of maintenance is a relevant aspect in the assessment of any industrial product or process, and therefore a challenging research problem. Our survey shows that maintenance approaches are continuously evolving over time. Earlier, corrective maintenance also known as reactive maintenance was used. Preventive maintenance proves to be a better alternative, as the maintenance actions are employed before the failure occurs. This approach evolved into condition-based maintenance, where decisions are based on the evaluation of the machine status through inspections and measurements. Among all the existent approaches to maintenance, each of them varying in terms of efficiency and complexity, predictive maintenance seems to best fit the needs of a highly competitive industry setup, as argued by [1]. Predictive maintenance allows maintenance actions to be based on changes of the machine and process parameters, which are continuously monitored by sensors. Currently, due to recent advances in sensor technology, data communication, and computing, the ability to collect big volumes of

This work was partly supported by a grant from the German Federal Ministry for Economic Affairs and Energy (BMWi) for the Platona-M project under the grant number 01MT19005D. heterogeneous, raw sensor data produced by equipment under observation is exponentially increasing. Therefore, historical information about normal and abnormal patterns and the related corrective actions employed during the lifetime of an industrial asset is becoming available. In order to deal with such high-dimensional problems, the predictive maintenance strategy uses a variety of techniques and prediction models that study both live and historical information. Further on, this information is used to learn prognostics data and to make accurate diagnostics and predictions, as presented by [2], [3], and [4]. They argue that the implementation of effective prognosis for maintenance has a variety of benefits including increased system safety, improved operational reliability, reduced maintenance, inspection times, repair failures and life cycle costs. Past works on predictive maintenance show that maintenance actions are performed by employing various prediction models and modeling techniques. Among prediction models, the machine learning (ML) approaches are typically considered the most suitable to deal with high dimensional and unstructured data, as argued by [8] and [9]. Moreover, multimodal fusion techniques are increasingly used by ML models for combining data from multiple, diverse modalities and sources with the goal of retrieving new insights from the fused knowledge i.e. multiple sensors may collect complementary or concurrent information which is fused in order to obtain more accurate machine diagnosis and prognosis. There is a lot of previous work on data fusion, as the topic dates back in the 90es. Application scenarios that implement ML models and apply multimodal data fusion for maintenance optimization purposes are defined by [2], [3], [9] and [10]. However, to date, no standard, nor good practice recommendations for fusion and integration of multimodal data have emerged. Our research work reviews the model-agnostic data fusion techniques in order to find solutions for their optimal usage. We argue that understanding the capabilities and challenges of existing multimodal data fusion methods and techniques has the potential to deliver better data analysis tools across all domains, including in the maintenance quality and management field of research. 


\section{A. Maintenance Issues Relative to Prediction Quality}

We envision the problematic of maintenance quality as a complex topic with many complementary aspects: economical, the conformity with the mainstream industrial standards and technical. The first aspect follows the classical optimization concerns relative to maintenance costs, by considering aspects related to maintenance investment costs and resulting benefits.

Traditional approaches consider maintenance only as cost related. However, the maintenance activities have direct implications to the production and quality, therefore should be treated as an investment, as argued by [11]. Moreover, appropriate timing for performing maintenance activities has economical justifications, as explained by [12] in the description of the damage model. The damage model recommends the usage of maintenance actions only when clear evidence about the machine or equipment status exists. It shows that based on the long-term, historical data, it is possible to adapt the predictive maintenance interval to the industrial item life cycle by forecasting the items wear, and the impact of it on the production chain, respectively. Reference [12] explains that the probability of an item to fail is high at the beginning of its operational life, in its burn-in period. During the burn-in period, the failure probability of an item is constantly decreasing. During the items working period, the failure probability is low and remains constant, therefore the prediction of the items failure during the working period is challenging. The probability of failure raises with the working hours so that in the wear period the probability for an item to fail is again high. Therefore, [12] recommends as a good practice to perform maintenance actions during the wear period of an items life cycle.

The second aspect which, we believe, influences the quality of maintenance is conformity with industrial standards during the development life cycle of a maintenance product. Our review of the literature shows the problematic of ad-hoc maintenance model development and implementations that do not comply with the existing mainstream standards. This situation leads to the absence of good practice recommendations or general solutions in the development of maintenance products. We briefly review two existing industrial standards for model development: Cross Industry Standard Process for Data Mining (CRISP-DM) and Industry Data Space [13]. CRISPDM standard represents a guideline to follow in the process of prototyping a learning model for maintenance purposes. We shortly list the guideline steps i.e. business understanding, data understanding, data preparation, data fusion, model prototyping, model evaluation, and deployment. A complete description is provided by the reference [45]. On its turn, Industry Data Space standard represents the solution to the actual problems raised by the huge volume of heterogeneous data which need to be handled in a standardized way in the industrial setup, as defined by [13]. Among the expected benefits of any standard, we mention the knowledge sharing and re-use which helps building complex, operational models.

The technical aspect of maintenance quality is related to the set of decisions concerning the appropriate techniques and approaches that should be used for the development of an operational and highly qualitative maintenance model. Our literature survey mainly focuses on analyzing the technical aspect, but it considers also its connections with the economic aspect. To our knowledge, none of the reviewed research works takes into account the conformity with industrial standards for model development and data management and security. One of the main issues of actual maintenance techniques and methods is exactly the absence of this holistic view in considering the problem of the maintenance quality as directly influenced by all the above three mentioned aspects. The rest of the paper is structured as follows: Section 2 starts with a review of maintenance approaches, according to the terminology defined by both [14] and [15] maintenance standards. We introduce a taxonomy that covers the surveyed approaches by categorizing the employed predictive models, the corresponding modeling techniques and the implementation algorithms, respectively. Further on, we review the literature works focusing on the technical steps of a maintenance model development process: data acquisition and analysis, data fusion, model development and evaluation, each of them being discussed in a subsection. Moreover, we present the concept of multimodal data fusion and we discuss a taxonomy of model-agnostic data fusion methods and their usage recommendations. Section 3 presents the review process we followed in gathering the literature for our survey. Findings and results of the investigated approaches are highlighted in Section 4. Finally, Section 5 concludes the paper with a discussion about the research challenges and future works.

\section{BACKGROUND}

\section{A. Classification of Maintenance Approaches}

The European recognized maintenance standards: DIN EN 13306 - Maintenance Terminology [14], and DIN EN 31051 - Fundamentals of Maintenance [15] are defining the maintenance related terminology and concepts. According to the DIN EN 31051 standard, the maintenance concept is defined as: the combinations of all technical and administrative actions as well as actions of management in the lifetime of a unit, in order to be in the fully functional state or to recover in this one, so that this unit can fulfill his requirements.

The main maintenance activities i.e. service, inspection, repair, and improvement are defined by the DIN EN 31051 standard. Their definitions together with other relevant maintenance concepts defined by the DIN EN 31051 maintenance standard are listed in Table I. The DIN EN 13306 maintenance standard defines the existing maintenance strategies: corrective maintenance, preventive maintenance, condition-based maintenance, and predictive maintenance. They are discussed in the following subsections. Moreover, the definition of a further maintenance strategy, namely prescriptive maintenance - which is not yet standardized, but already used in practice is discussed in the following subsection. 
TABLE I

FUNDAMENTALS OF MAINTENANCE DIN EN 31051 STANDARD

\begin{tabular}{|c|c|}
\hline Item & Defines a component, device, subsystem, functional unit, equipment or a system which can be described and considered as an entity. \\
\hline Wear & Represents the reduction of wear margin due to chemical or physical processes. \\
\hline Wear limit & Is the defined minimum value of the wear margin. \\
\hline Wear margin & Defines the possible reserve function capacity under defined circumstances which a unit possesses. \\
\hline Inspection & $\begin{array}{l}\text { Refers to all activities used to determine and evaluate the actual conditions of facilities, machines, assemblies or components. } \\
\text { Inspection refers to collecting data, and related activities that can be measuring, verifying and monitoring. }\end{array}$ \\
\hline Repair & Covers activities for retrieving the nominal condition, such as renewing, patching and adjusting. \\
\hline
\end{tabular}

1) Corrective Maintenance: According to the EN 13306 standard, the corrective maintenance is defined as the maintenance carried out after fault recognition and intended to put an item into a state in which it can perform a required function. A system that employs corrective maintenance is aware of all its predefined set of failures and damages. But, in the industrial, operational context new faults and their corresponding patterns appear over time, because of the items usage during the working hours. One main advantage of applying corrective maintenance techniques is that the wear-limit of an item, i.e. the service time is fully used. This implies that the effort for items inspection and for replacing the item is significantly reduced, compared with the case of preventive maintenance. The main challenge in applying corrective maintenance is that the item can fail at an unknown time not previously known or decided and consequently can produce damages and an additional cost that can be higher as the yield of full usage of its wear margin.

2) Preventive Maintenance: The EN 13306 standard defines preventive maintenance as the maintenance carried out at predetermined intervals or according to prescribed criteria and intended to reduce the probability of failure or the degradation of the functioning of an item. One main challenge of preventive maintenance in operational context is that industrial scenarios for data analysis do not provide tracking of the past, abnormal behavior or maintenance operations that were performed in order to correct or to prevent a faulty behavior. Consequently, preventive maintenance defines a set of actions carried out before failure and that are intended to prevent failures or degradation of a machine. Time-based maintenance is defined as the preventive maintenance approach that recommends performing all maintenance activities after a certain amount of operation hours, or by predefined scheduling, regardless of the items health condition. The assumption is that after several operational hours, the wear margin of an item is worn out. The employed approach is to change the item or to overhaul part of it before the wear margin is used. The advantages of time based maintenance are the reduced breakdown frequency and the increased service life compared with other preventive maintenance strategies. It is therefore recommended only when the safety of the environment can be harmed, or when the items lifetime is known, which is not the case in the operational environment. The economic justi- fication behind the time-based maintenance approach is that the maintenance costs can be kept low when the maintenance interval is adjusted to the actual lifetime of the asset so that the item or some of its parts are changed just before they fail.

3) Condition-based Maintenance: The EN 13306 standard defines condition-based maintenance as the preventive maintenance which includes a combination of condition monitoring and/or inspection and/or testing, analysis, and the ensuing maintenance actions. Condition-based maintenance aims to anticipate a maintenance operation based on the evidence of degradation and deviations from a supposed asset normal behavior. The equipment is monitored with multiple sensors which are supposed to acquire relevant data about the equipment operation life. Additionally, contextual information like temperature, humidity, etc. may also provide significant information. Key Process Indicators (KPIs) or health indicators are usually computed and analyzed, in order to discover trends that lead to abnormal contexts and failure events.

4) Predictive Maintenance: According to the EN 13306 standard, predictive maintenance is defined as the conditionbased maintenance carried out following a forecast derived from repeated analysis or known characteristics and evaluation of the significant parameters of the degradation of the item. Predictive maintenance is a sub-class of condition based maintenance. It uses a variety of approaches and ML techniques to study both recent and historical data and to learn prognostic models which are expected to make accurate predictions about the future status of a machine or equipment. The main challenge of predictive models is that they rely on the assumption that there are certain contexts in the equipment life time where the failure rate is increasing. In the industrial, operational context there are patterns in which the failure probability does not increase, but remains constant during the equipment life time, and therefore the equipment can fail at any time: it is the case of electrical and electronic components.

5) Prescriptive Maintenance: Terminologically, it is mentioned by neither the EN 13306, nor the DIN EN 31051 maintenance standards. But, its functionality can be consequently deduced and is seen as a recommendation of one or more courses of action based on the outcomes of models for corrective and predictive maintenance. The main challenge of prescriptive maintenance is the difficulty to build in practice operative models. Existing research models are based on ad- 
hoc model development where ML methods and data fusion techniques are jointly used with fuzzy reasoning, simulation techniques, and evolutionary algorithms.

Tables II, III and IV introduced in Section 4 are constructed based on the reviewed literature on maintenance strategies i.e. corrective, preventive and predictive. The tables present the surveyed literature, i.e. a structured review of the maintenance type and goals, correlated with a specific statistical or datadriven operational method, and the corresponding results. For a better understanding of implementation techniques for maintenance purposes, the next section reviews the basic steps of a data-driven model development life cycle i.e. data acquisition and preparation, model development (including the multimodal ML methods discussion) and model evaluation.

\section{B. Data Driven Model Development Life Cycle Methods and Techniques for Maintenance Purposes}

Understanding the specific application context, or the business requirements is the first step for any learning model developed and deployed in an industrial environment. The basic steps of a data driven model development life cycle for maintenance purposes are discussed in the next subsections.

1) Data Acquisition and Preparation: Predictive models learn patterns from historical, multimodal data and predict future outcomes with certain probability based on these observed patterns. The performance of any learning model is highly correlated with the relevancy, sufficiency, and quality of the training, validation and test data. Data pre-processing and feature extraction techniques are relevant in building reliable data driven models. Processing the raw data before modeling is improving the performance of the learned model. In practice, raw data in the form of sensor signals are complex and related information about the degradation process of the monitored component is not always available. Therefore, preprocessing raw sensor data is a mandatory step before building the maintenance models. Generally, data processing methods can be divided into two main tasks, namely processing and data analysis.

2) Model Development: In the context of a data-driven model development life cycle, the ML techniques for maintenance is considered the most suitable research perspective to deal with big volumes of heterogeneous data. ML techniques comprise two main approaches: (i) supervised learning, where the information about the occurrence of failures is present in the modeling data set; and (ii) unsupervised learning, where only the process information is available and no historic maintenance data exists. In an operational environment, predictive maintenance makes use of the following well-established supervised learning techniques from ML field: (i) classification algorithms which are used to represent groups of normal and abnormal health status of the item under observation i.e. Random Forest, Nearest Neighbors, SVMs and HMMs; (ii) regression algorithms; and (iii) clustering methods with anomaly detection algorithms. Multimodal machine learning (MML) represent an increasingly used set of ML methods for combining data from multiple and diverse modalities and sources with the goal of retrieving new insights from the combined knowledge i.e. multiple sensors collect complementary or concurrent information which is combined in order to obtain more accurate machine diagnosis and prognosis. We provide in Section 4 an overview of the multimodal ML methods which shows that the multimodal fusion method seems to be the most employed for maintenance goals.

3) Model Evaluation: Once a model is built, an estimate of its performance is required. According to [41] there are two types of evaluation metrics that are giving insights about the quality of the model performance metrics: offline evaluation metrics that measure offline data of the prototype model and uses mainly historic data, and online evaluation metrics that measure live metrics on the deployed model on real-time data. Offline evaluation is used to estimate the performance of training and validating data, and therefore performance metrics like accuracy and precision-recall together with F1Score are employed. An online evaluation usually is used for real-time, test data e.g. to estimate business metrics. Our survey focuses on reviewing the offline evaluation metrics used in industry for evaluation learning models for prediction maintenance purposes. The model evaluation is made on a different set of data, i.e. testing data set that is statistically independent of the data set that it was previously trained on. Mathematically speaking, the model evaluation means to estimate the generalization error of the learning model, i.e. to measure how good the model behaves under new data calibrations. A good practice is to split the data set into training, validation and test data, in a time dependent manner. Further good practice is to consider the training data earlier in time than all the validation and test data. Andrew $\mathrm{Ng}$ recommends a split such as training set (60\%), cross-validation-set (20\%) and testing set $(20 \%)$. The confusion table (also called confusion matrix) it is used to show a detailed breakdown of correct and incorrect classifications and is applied for evaluating models that learn from highly imbalanced data. Performance metrics based on the confusion table are accuracy, precision, recall, specification, F1-Score, the AUC-ROC Curve.

\section{RESEARCH APPROACH}

The aim of this research review is to increase knowledge in the field of maintenance techniques and their operationalization. This implies a sort of awareness in considering the appropriate predictive models and their corresponding implementation techniques depending on the available data and on the application scenario. The conference and journal publications selected for our review belong to the non-empirical conceptual and mathematical field of research. Consequently, they describe issues and perspectives related to maintenance strategies and their modeling techniques applied in an industrial setup. A systematic search using online databases was employed for a keyword-based search, in order to find journal and proceeding publications. We used the English language and the following keywords: maintenance AND machine learning. We iteratively continued the search using the following keywords: predictive 
maintenance, multimodal machine learning, multimodal fusion, multimodality, maintenance AND big data, maintenance AND Industry 4.0. We finally obtained a shorter literature list which was further reduced by eliminating the duplicates, when similar topics and approaches were found. Science Direct, Scopus and Google Scholar literature databases were used for their wide coverage of journals, proceedings and books.

\section{A. Description of the Criteria Used for Analysis}

Our research perspective relative to the maintenance quality problematic focuses on: (i) the decision process to choose a specific maintenance approach i.e. maintenance goals, benefits, challenges and obtained results; and (ii) the implementation of the maintenance approach i.e. the employed prediction models and their corresponding modelling techniques. The selected literature was carefully examined in order to extract useful information based on the following criteria:

- Prediction models reveal a taxonomy of the most employed prediction models types employed in a maintenance process i.e. physical models, knowledge-based models, databased models and hybrid models.

- Modelling techniques represent the implementation pipeline (data analysis + algorithms) used. It is a relevant criterion which further helps us to select the set of the most used ML algorithms to be critical reviewed.

- Dataset comprises information about the involved sensor types and the kind of fusion applied. It is a relevant criterion which further helps us to provide a critical analysis of the quality of data involved in a maintenance process.

- Industry is concerned with the branch of industry where maintenance processes are applied.

- Equipment parts reveal the critical parts of equipment which are considered for maintenance.

- Obtained results /performance metrics extract the information concerning how the model was evaluated and give us a hint about how optimal the data analysis and learning algorithms were applied.

- Maintenance goals provide us with a taxonomy of topics showing the final decisions of the algorithms pipeline. Paired with the Modelling techniques criterion, it gives useful information about the successful algorithm pipeline used for a certain maintenance goal.

The overview of the reviewed maintenance literature is presented in Tables II, III and IV. We are not considering for our research works the empirical perspective, i.e. we are not discussing the maintenance strategies and their operationalization based on information obtained from interviews, or from analyzing case studies. The analytic literature review we conduct is formalized by [17] and [18] and starts with clarifying relevant maintenance terminology and definitions based on the accepted, European maintenance standards [15] and [14].

\section{Findings And Results}

This section presents the reviewed results displayed in Tables II, III and IV. The surveyed works we consider are grouped by maintenance type, and further on they are grouped by prediction modeling types and relevant modeling techniques used in the implementations.

\section{A. Analysis of Maintenance Strategies}

1) Corrective Maintenance: Our survey shows that the fault recognition and diagnostic is generally seen as a process of pattern recognition i.e. the process of mapping the information i.e the features obtained in the measurement space to the machine faults in the fault space, as described in [19], [20], [21] and [22]. Diagnosis is a necessary part of any maintenance system, as only prognostics cannot provide in practice a sure prediction which covers all failures and faults. In case of unsuccessful prognosis, a diagnosis is a complementary tool for providing maintenance decision support. The methods employed in order to deal with fault classification and diagnostics are diverse: from expert systems [23] to Hidden Markov Models (HMM)s, as presented in [19], Artificial Neural Networks (ANN)s as described in [20], Support Vector Machine (SVM) as in [21] and fuzzy algorithms enhanced with spectral clustering and Haar wavelet transform, as described in [22].

2) Preventive Maintenance: The reviewed literature shows that a relevant class of preventive maintenance techniques are the prognostics through pattern recognition, classification and machine health status identification. Prognostics analyze data by automatically finding new insights in terms of behavioral patterns. The information extracted from the monitored data can help detecting patterns that characterize the machine working conditions or is anticipating and estimating critical events i.e. fault detection as in [3] and Remaining Useful Life (RUL) estimation as in [8]. Prognostics are considerate superior to diagnostics in the sense that they prevent faults and are employed for prediction problems with items spare parts and human resources, saving unplanned maintenance costs. The reference [5] proposes a data mining maintenance approach for predicting material requirements in the automotive industry by measuring the similarity of customer order groups. Identifying behavioral patterns in data means to classify similar data in some data-groups which share the same characteristics i.e. operational conditions, as described by [24], [25], [26], [27] and [28]. Within these classified groups there are data-points that are far from the identified pattern i.e. the outliers, or they may correspond to a distinctive property i.e. the mean point or the group distribution. Such patterns may help to identify faults or any other type of abnormal behavior. Large groups of data are interpreted as normal behavior, while small groups of data, or events that are far from the pattern are usually representing anomalies. Consequently, in modeling the learning model, there are only unlabeled input examples, i.e. we employ the the unsupervised learning perspective. ML algorithms and data fusion strategies are used to find new patterns in data therefore, in this case, clustering should be the most used technique, 
TABLE II

REVIEW OF CORRECTIVE MAINTENANCE MODELS AND CORRESPONDING IMPLEMENTATION TECHNIQUES

\begin{tabular}{|c|c|c|c|c|c|c|c|}
\hline References & $\begin{array}{c}\text { Prediction } \\
\text { models }\end{array}$ & $\begin{array}{l}\text { Modeling } \\
\text { techniques }\end{array}$ & Dataset(s) & Industry & $\begin{array}{c}\text { Equipment } \\
\text { parts }\end{array}$ & $\begin{array}{l}\text { Obtained results } \\
\text { e.g. performance metrics }\end{array}$ & $\begin{array}{l}\begin{array}{c}\text { Maintenance } \\
\text { goals }\end{array} \\
\text { gols }\end{array}$ \\
\hline \multirow{5}{*}{$\begin{array}{l}\text { (Bunks, C., et al., 2004) } \\
\text { [19] } \\
\text { (Deuszkiewicz, P., et al.; 2003) } \\
\text { [20] } \\
\text { (Hao, Y., et al.; 2005) } \\
\text { [21] } \\
\text { (Baraldi, P., et al.; 2014) } \\
\text { [22] } \\
\text { (Alexandru, A., 1998) } \\
\text { [23] }\end{array}$} & \multirow[t]{2}{*}{$\begin{array}{l}\text { Knowledge } \\
\text { based models }\end{array}$} & $\begin{array}{l}\text { Expert Systems + } \\
\text { fault tree analysis }\end{array}$ & $\begin{array}{l}\text { functional sensor data } \\
\text { of the PV pilot plant }+ \\
\text { meteorological sensor data }\end{array}$ & $\begin{array}{l}\text { Water pumping } \\
\text { station }\end{array}$ & $\begin{array}{l}\text { PV pilot plant Zambelli, } \\
\text { Italy (Joule II EU Project) }\end{array}$ & $\begin{array}{l}\text { real time supervision and monitoring }+ \\
\text { detection of foreseen faults }\end{array}$ & $\begin{array}{l}\text { real time monitoring; } \\
\text { maintenance inspection } \\
\text { on request }\end{array}$ \\
\hline & & $\begin{array}{l}\text { Fuzzy similarity, } \\
\text { fuzzy c-means } \\
\text { algorithm }\end{array}$ & $\begin{array}{l}\text { synthetic data of simulated faults } \\
\text { in a pressurizer water reactor (PWR) } \\
\text { NPP: vapor and steam temperature, liquid } \\
\text { temperature, liquid level and pressure }\end{array}$ & $\begin{array}{l}\text { Nuclear Power } \\
\text { Plant }\end{array}$ & Pressurizer water system & $\begin{array}{l}\text { drawback: new faults cannot be } \\
\text { classified into new groups without } \\
\text { repeatedly applying the spectral analysis }\end{array}$ & $\begin{array}{l}\text { classification models } \\
\text { for fault diagnosis } \\
\text { using unsupervised } \\
\text { clustering }\end{array}$ \\
\hline & \multirow[t]{3}{*}{$\begin{array}{l}\text { Data based } \\
\text { models }\end{array}$} & $\begin{array}{l}\text { Stochastic model: } \\
\text { Hidden Markov } \\
\text { Models (HMMs) }\end{array}$ & $\begin{array}{l}\text { vibration measurements from a set of } \\
8 \text { accelerometers from the gearbox, } \\
\text { at } 9 \text { torque levels and } 8 \text { seeded } \\
\text { defects }\end{array}$ & Naval Research & $\begin{array}{l}\text { Westland helicopter } \\
\text { gearbox }\end{array}$ & $\begin{array}{l}\text { HMMs are fully probabilistic models } \\
\text { incorporating quasi-stationarity } \\
\text { as a feature + build robust and flexible } \\
\text { classification models }\end{array}$ & $\begin{array}{l}\text { machine health } \\
\text { status diagnostics; } \\
\text { defect type } \\
\text { classification }\end{array}$ \\
\hline & & $\begin{array}{l}\text { Artificial } \\
\text { Neural Networks } \\
\text { (ANNs) }\end{array}$ & $\begin{array}{l}\text { sensors from the body of the driving } \\
\text { axle box at various speed ( } 50,70,90, \\
110 \mathrm{Km} / \mathrm{h} \text { ), crest factor signal, XSK signal }\end{array}$ & $\begin{array}{l}\text { Railway } \\
\text { (ZNTK S.A. } \\
\text { Rolling Stock } \\
\text { Repair Company) } \\
\end{array}$ & $\begin{array}{l}\text { Power transmission } \\
\text { unit in (ED-72 train) } \\
\text { rolling bearing }\end{array}$ & $\begin{array}{l}\text { minimizes the frequency of revision } \\
\text { inspections }+ \text { in time online warning for } \\
\text { unexpected new failures }\end{array}$ & $\begin{array}{l}\text { Machine health } \\
\text { status diagnostics } \\
\text { in useful time }\end{array}$ \\
\hline & & $\begin{array}{l}\text { Statistical model: } \\
\text { Support Vector } \\
\text { Machine (SVM)+ } \\
\text { k-fold cross validation }\end{array}$ & $\begin{array}{l}\text { gas temperature, fuel flow, pressure } \\
\text { rotor speed }\end{array}$ & Aerospace & Gas Turbine engine & $\begin{array}{l}\text { accuracy: } 93 \% \text { even when the } \\
\text { standard deviation of noise is } 3 \text { times } \\
\text { larger than normal: a better } \\
\text { generalization than ANNs }\end{array}$ & $\begin{array}{l}\text { Identification of } 3 \\
\text { most possible faults } \\
\text { types }\end{array}$ \\
\hline
\end{tabular}

TABLE III

REVIEW OF PREVENTIVE MAINTENANCE MODELS AND CORRESPONDING IMPLEMENTATION TECHNIQUES

\begin{tabular}{|c|c|c|c|c|c|c|c|}
\hline References & $\begin{array}{c}\text { Prediction } \\
\text { models }\end{array}$ & $\begin{array}{l}\text { Modeling } \\
\text { techniques }\end{array}$ & Dataset(s) & Industry & $\begin{array}{c}\text { Equipment } \\
\text { parts }\end{array}$ & $\begin{array}{c}\text { Obtained results } \\
\text { e.g. performance metrics }\end{array}$ & $\begin{array}{c}\text { Maintenance } \\
\text { goals }\end{array}$ \\
\hline \multirow{6}{*}{$\begin{array}{l}\text { (Manco, G., et al.; 2017) } \\
\text { [3] } \\
\text { (Krishnakumari, A., et al.; 2017) } \\
\text { [24] } \\
\text { (Jaramillo, V.H., et al.; 2017) } \\
\text { [25] } \\
\text { (Liu, C., et al.; 2016) } \\
\text { [26] } \\
\text { (Diez, A., et al.; 2016) } \\
\text { [27] } \\
\text { (Li,C., et al.; 2016) } \\
\text { [28] }\end{array}$} & $\begin{array}{l}\text { Hybrid } \\
\text { models }\end{array}$ & Outlier detection & $\begin{array}{l}\text { failures, events described by } \\
\text { type, timestamp, subsystems, } \\
\text { duration, severity, description }\end{array}$ & Railway & Train doors & $\begin{array}{l}\text { High degree outliers are effective } \\
\text { indicators of incipient failures. }\end{array}$ & fault detection \\
\hline & $\begin{array}{l}\text { Knowledge } \\
\text { based models }\end{array}$ & $\begin{array}{l}\text { Fuzzy } \\
\text { Classifier + } \\
\text { Decision Tree }\end{array}$ & $\begin{array}{l}\text { Feature extraction + monitored } \\
\text { data representing condition-based } \\
\text { status of vibration signals }\end{array}$ & Manufacturing & $\begin{array}{l}\text { Gears in rotary } \\
\text { machines }\end{array}$ & $\begin{array}{l}\text { Feature extraction and classification } \\
\text { explained. The performance of the } \\
\text { fuzzy inference has } 95 \% \text { accuracy. }\end{array}$ & $\begin{array}{l}\text { pattern recognition + } \\
\text { fault detection and } \\
\text { classification }\end{array}$ \\
\hline & \multirow{4}{*}{$\begin{array}{l}\text { Data based } \\
\text { models }\end{array}$} & $\begin{array}{l}\text { Statistical model: } \\
\text { Bayesian } \\
\text { Inference }\end{array}$ & $\begin{array}{l}\text { Multi sensor feature based } \\
\text { fusion (acceleration, current, } \\
\text { voltage, temperature) }\end{array}$ & Manufacturing & $\begin{array}{l}\text { Electric motor } \\
\text { with two gearboxes } \\
\text { and a load }\end{array}$ & $\begin{array}{l}\text { Feature based fusion + concepts of } \\
\text { global/local fusion + feature extraction } \\
\text { is good explained based on the example } \\
+ \text { transparent Bayesian inference method }\end{array}$ & $\begin{array}{l}\text { machine health } \\
\text { status assessment } \\
\text { and condition } \\
\text { monitoring }\end{array}$ \\
\hline & & $\begin{array}{l}\text { Statistical model: } \\
\text { SVM +Fourier } \\
\text { transform + discrete } \\
\text { Wavelet decomposition }\end{array}$ & $\begin{array}{l}\text { Multisensor feature-based } \\
\text { fusion (dynamometer sensor, } \\
\text { acceleration sensor, cutting force, } \\
\text { vibration signal) }\end{array}$ & Manufacturing & $\begin{array}{l}\text { Cutting tools } \\
\text { and flank } \\
\text { milling machines }\end{array}$ & $\begin{array}{l}\text { Accuracy: } 90 \% \text { information feature- } \\
\text { based fusion with multiple sensors } \\
\text { provide complementary information } \\
\text { to machining conditions }\end{array}$ & $\begin{array}{l}\text { multiple machine } \\
\text { condition monitoring } \\
\text { and recognition }\end{array}$ \\
\hline & & $\begin{array}{l}\text { k-NN based outlier } \\
\text { remover + clustering } \\
\text { approach of vibration } \\
\text { events and joints }+ \\
\text { Fourier transform }\end{array}$ & $\begin{array}{l}\text { Multi-sensor feature-based } \\
\text { fusion (accelerometer sensor }+ \\
\text { location sensor) }\end{array}$ & Construction & $\begin{array}{l}\text { Bridges (Sydney } \\
\text { Harbour Bridge) }\end{array}$ & $\begin{array}{l}\text { Real time health score (of the structure) } \\
\text { learned from historical data and used to } \\
\text { check new events based on cluster } \\
\text { centroids and joints representatives. }\end{array}$ & $\begin{array}{l}\text { damage detection } \\
\text { of abnormal or } \\
\text { damaged }\end{array}$ \\
\hline & & $\begin{array}{l}\text { ANN and } \\
\text { Deep Learning }\end{array}$ & $\begin{array}{l}\text { Automatic multisensor feature } \\
\text { fusion from vibration, signal } \\
\text { measurements }\end{array}$ & Manufacturing & Rotary machines & $\begin{array}{l}\text { Deep Learning with statistical } \\
\text { feature representation shows better } \\
\text { performance metrics. Statistical features } \\
\text { in the time, frequency and time-frequency } \\
\text { domains have different representation } \\
\text { capabilities for fault patterns. }\end{array}$ & $\begin{array}{l}\text { fault diagnostic } \\
\text { and fault patterns } \\
\text { identification }\end{array}$ \\
\hline
\end{tabular}

together with a measure of similarity which should deal with showing the correspondence of data groups. When pattern classification is applied for describing training data, then we assume the availability of (i) historic data with abnormal behavior; and (ii) data concerning maintenance activities that were carried out. The learner looks for identifying the causes for confirmed, abnormal behavior and critical events, in order to predict them and to avoid them in the future. In this case, values for the target labels are available, and therefore the supervised learning strategy and the corresponding algorithms are to be employed. The target labels are representing features that are discrete or continuous, and they are always related to the diagnostic. Time-series analysis is employed to extract damage and fault-sensitive features from data. When a corrective action is made, the preceding data represent an abnormal behavior or abnormal data context. When events of interest or based on past maintenance actions are tracked, they are assumed to represent a normal data context. Another type of scenario is learning the normal behavior of the machine or parts from its equipment. This is a difficult process in the operational context, as it supposes that there are no outliers, nor operational faults, which is not the case of the industrial environment. Finding patterns in the monitored data requires a deep knowledge of the topic and of physics of the process so that the issue can be theoretically understood. In the context of supervised learning approaches, feature engineering and mainly the interpretation of the assessment of the results represent always a challenge.

3) Predictive Maintenance: The survey shows that the predictive maintenance process has the goal of providing an accurate estimate of the RUL, but also it should asses the provided estimate, as argued in [31], [32], and [33]. Time-series analysis is used to anticipate anomalies and malfunctions in equipment and processes maintenance procedures. Traditional approaches are moving average over a time window, ARMA/ARMAX, Kalman Filter and cumulative sum, as described in [9]. Recursive Neuronal Networks (RNNs) show relevant characteristics for time series forecasting, as their loops allow information to persist, as presented in [8]. Multi sensor fusion ranges from multi signal combinations, as argued in [8] and [9], to more complex integration of conditional assessment, RUL estimation, and decision making, as presented in [2] and [10]. Operational predictive approaches are based on a schema that implies frequent, and sometimes unnecessary maintenance of the equipment and of the entire production process that leads to high maintenance time and costs. They use complex A.I. based algorithms, and data fusion strategies - in an ad-hoc manner, usually after trial and error approaches - which imply the usage 
of consecutive fusion algorithms, as described by reference [26]. The uncertainty in prediction is always a challenge and to this time the fuzzy logic is used to represent uncertainties in prediction, as argued by [4]. As a particular case of conditionbased maintenance, reference [29] shows that techniques for condition monitoring and diagnostics are gaining acceptance in the industry sectors, as they prove to be effective also in the predictive maintenance and quality control areas. The authors apply a feature based fusion technique implemented with the cascade correlation neuronal network to multiple sensor data collected from rotating imbalance vibration of a test rig. The results show that the multi-sensory data fusion outperforms the single sensor diagnostic. The reference [30] focuses on the capability of providing real-time maintenance by extracting knowledge from the monitored assets (with vibration sensors) on the production line. Using intelligent data driven monitoring algorithms (ADMM), data fusion strategies and the proposed three-levels layered (IoT, Fog with gateway nodes for sensors aggregation, Decision) system model, the authors argue on the efficiency of cloud oriented maintenance.

The uncertainty in prediction is always a challenge and to this time the fuzzy logic is used to represent uncertainties in prediction, as argued by [4].The references [6] and [7] show that the problem of scheduling under constraint of completion time of all production jobs can also be solved using predictive maintenance algorithms. The efficiency of the algorithms for predicting machine failures is further evaluated using simulation tests. The results, i.e. the optimized jobs schedule shows a nearly $50 \%$ drop in the number of operations compared with the initial, nominal schedule. The classification of modeling techniques for predictive models is presented in Figure 1. Physical models use the laws of physics to describe the behavior of a failure [2]. Knowledge-based models assess similarities among observed situations and a set of previously defined failures. These models can be sub-divided in expert system models able to answer complex queries, as presented by [23], and fuzzy models, as in [4]. Data-driven models are based on the acquired data. This type of model can distinguish among stochastic models, statistical models and artificial neural networks (ANNs). Hybrid models use combinations of two or more modeling techniques as in [34], [35] and [44]. Stochastic models provide event-based information. Hidden Markov models and Kalman filters belong to this category too. Statistical models predict a future state by comparing the monitored results with a machine health state without faults. ML models, such as regressions, classifications, and clustering represent a category of data-based, statistical models relevant in the study of maintenance optimization. However, the ML models are focusing on increasing the accuracy of their predictions, while the classical statistical community is more concerned with the understanding of their models and of the model's parameters i.e. model calibration and inference.

4) Prescriptive Maintenance: The main challenge of prescriptive maintenance is the difficulty to build in practice operative models. Existing research models are based on adhoc model development where ML methods and data fusion techniques are jointly used with fuzzy reasoning, simulation techniques, and evolutionary algorithms. The reviewed literature shows that prescriptive maintenance implementations show an ad-hoc grouping of methods including data fusion and ML techniques, combined with fuzzy reasoning algorithms, simulations [34] and multi-objective evolutionary algorithms for optimization [44]. When a predictive model raises an alarm before the fault occurs, the prescriptive model will work in the direction of reducing the probability that this alarm will rise in the future, by modifying the working parameters and variables of the asset or the process affected by the fault. When the fault is confirmed, the prescriptive models will work to minimize its impact of the work context and to re-routing assets to the non-faulty production lines.

The tables II, III and IV are constructed based on the reviewed literature on maintenance types: corrective, preventive and predictive. The tables present a structured view of the maintenance type and goals, correlated with a specific statistical or data-driven operational method, and the corresponding results.

\section{B. Analysis of Data Driven Development Life Cycle}

1) Data Acquisition and Preparation: Predictive models learn patterns from historical, multimodal data and predict future outcomes with certain probability based on the observed patterns. The performance of any learning model is highly correlated with the relevancy, sufficiency, and quality of the training, validation and test data. Moreover, the data used for training and testing the model should be relevant for the application scenario, therefore the expertise and the guidance of a domain expert is important. The most relevant data sources for a predictive model application scenario are condition monitoring data referred to as hard data, and human generated data referred to as soft data. Condition monitoring data contains knowledge in the form of degradation patterns and other types of anomalies in data that leads to an item degradation. Time-varying features are expected to capture these abnormal patterns, and the models fed with these features are expected to learn to distinguish between normal and abnormal pattern behaviors of items and also to forecast the RUL for the monitored items. Condition monitoring data can be further decomposed into sensor data, asset data, operation data, offline inspection data, and historical data. On its counterpart, human generated data represents information about replaced components, repair activities performed on a certain item or on parts of it. Moreover, it consists also of software generated information e.g. event data information such as alarms and faults messages which are described in natural language, but it comprises also technical metadata for devices and processes i.e. model, manufactured date, the start of service, maintenance reports. The event-data collection implies always a manual process and includes qualitative information about the monitored item such as the description of the installation, breakdown, inspection, repair, overhaul, failure causes, etc., the severity of the failure and the description of what was done to fix the failure. In practice, the item under critical 
TABLE IV

REVIEW OF PREDICTIVE MAINTENANCE MODELS AND CORRESPONDING IMPLEMENTATION TECHNIQUES

\begin{tabular}{|c|c|c|c|c|c|c|c|}
\hline References & $\begin{array}{c}\text { Prediction } \\
\text { models }\end{array}$ & $\begin{array}{c}\text { Modeling } \\
\text { techniques } \\
\end{array}$ & Dataset(s) & Industry & $\begin{array}{c}\begin{array}{c}\text { Equipment } \\
\text { parts }\end{array} \\
\end{array}$ & $\begin{array}{c}\text { Obtained results } \\
\text { e.g. performance metrics } \\
\end{array}$ & $\begin{array}{c}\text { Maintenance } \\
\text { goals }\end{array}$ \\
\hline \multirow{8}{*}{$\begin{array}{l}\text { (Xenakis, A., et al.; 2019) } \\
\text { [30] } \\
\text { (Liu, Z., et al.; 2018) } \\
\text { [2] } \\
\text { (Niu, G., et al.; 2017) } \\
\text { [4] } \\
\text { (Guo, L., et al.; 2017) } \\
\text { [8] } \\
\text { (Acorsi, R., et al.; 2016) } \\
\text { [9] } \\
\text { (Mosallam, A., et al.; 2016) } \\
\text { [31] } \\
\text { (Cristaldi, I., et al.; 2016) } \\
\text { [33] } \\
\text { [Safizadeh, M., et al.; 2014) } \\
\text { [10] }\end{array}$} & $\begin{array}{l}\text { Knowledge } \\
\text { based models }\end{array}$ & $\begin{array}{l}\text { Rule-based fuzzy } \\
\text { logic }+ \text { condition-based } \\
\text { fusion diagnosis }\end{array}$ & $\begin{array}{l}\text { Multisensor decision level } \\
\text { fusion (vibration signal }+ \\
\text { current signal) }\end{array}$ & Railway & $\begin{array}{l}\text { Electric multiple units } \\
\text { (EMU) trains }>>\text { pulling } \\
\text { motor of EMU bogie }\end{array}$ & $\begin{array}{l}\text { the accuracy of multiple classifier fusion } \\
\text { (vibration/current features) is greater as } \\
\text { the accuracy of single classifiers }\end{array}$ & general maintenance \\
\hline & \multirow[t]{5}{*}{$\begin{array}{l}\text { Data based } \\
\text { models }\end{array}$} & $\begin{array}{l}\text { ADMM } \\
\text { (altering direction } \\
\text { method of multipliers) } \\
\text { algorithm }+ \\
\text { Decision Fusion }\end{array}$ & $\begin{array}{l}\text { 1-second vibration } \\
\text { signals snapshots } \\
\text { with the samplig } \\
\text { rate set at } 20 \mathrm{kHz}\end{array}$ & Industrial Automation & Production Line & $\begin{array}{l}\text { minimize operational costs }+ \\
\text { efficient energy consumption }\end{array}$ & $\begin{array}{l}\text { real time } \\
\text { analyse and } \\
\text { process of } \\
\text { machine faults }+ \\
\text { health status } \\
\text { monitoring }\end{array}$ \\
\hline & & $\begin{array}{l}\text { RNN-based health } \\
\text { indicator for RUL } \\
\text { prediction }\end{array}$ & $\begin{array}{l}\text { Multisensor fusion at feature } \\
\text { level (vibration signals }+ \\
\text { time-frequency features) }\end{array}$ & Aerospace & Bearings & $\begin{array}{l}\text { high RUL prediction accuracy } \\
\text { of generator bearings }\end{array}$ & $\begin{array}{l}\text { RUL } \\
\text { prediction }\end{array}$ \\
\hline & & $\begin{array}{l}\mathrm{kNN}+\text { discrete } \\
\text { Bayesian filter }\end{array}$ & $\begin{array}{l}\text { KIP from operational data } \\
\text { (NASA Lithium-Ion Battery } \\
\text { B0005->B0056 repository)+ } \\
\text { Turbofan engine data C-MAPSS }\end{array}$ & Aerospace & $\begin{array}{l}\text { Battery and } \\
\text { turbofan engine }\end{array}$ & $\begin{array}{l}\text { 3-fold cross validation is successfully } \\
\text { validating the approach. average MAPE } \\
\text { is computed and generates low errors } \\
\text { for both applications }\end{array}$ & $\begin{array}{l}\text { RUL } \\
\text { prediction }\end{array}$ \\
\hline & & $\begin{array}{l}\text { Statistics, } \\
\text { Deep Learning }\end{array}$ & $\begin{array}{l}\text { Features extraction from } \\
\text { single product and fleet levels }\end{array}$ & $\begin{array}{l}\text { Electrical Power } \\
\text { plants }\end{array}$ & $\begin{array}{l}\text { Medim/High } \\
\text { circuits breakers }\end{array}$ & $\begin{array}{l}\text { Health Condition Profile with RUL and } \\
\text { PoF (Probability of Failure) computed } \\
\text { in a predetermined window of time. }\end{array}$ & $\begin{array}{l}\text { RUL and } \\
\text { PoF prediction }\end{array}$ \\
\hline & & $\mathrm{PCA}+\mathrm{kNN}$ & $\begin{array}{l}\text { Multisensor fusion (waterfall } \\
\text { fusion technique) at feature level } \\
+ \text { decision level (accelerometer } \\
\text { data + load cell data) }\end{array}$ & Manufacturing & $\begin{array}{l}\text { Rolling } \\
\text { bearings }\end{array}$ & $\begin{array}{l}\text { Data from different sensors provide } \\
\text { more information than data gathered } \\
\text { from single ones. }\end{array}$ & $\begin{array}{l}\text { condition-based } \\
\text { monitoring and } \\
\text { diagnosis }\end{array}$ \\
\hline & \multirow[t]{2}{*}{$\begin{array}{l}\text { Hybrid } \\
\text { models }\end{array}$} & $\begin{array}{l}\text { k-means, association } \\
\text { rules (GSP, Apriori), } \\
\text { Neural Networks, } \\
\text { Random forest, } \\
\text { Decision Tree, kNN }\end{array}$ & $\begin{array}{l}\text { Feature extraction from } \\
\text { parameter logs (user settable } \\
\text { machine quantities), message } \\
\text { logs and energy data sampling } \\
\text { sensors }\end{array}$ & Manufacturing & $\begin{array}{l}\text { Automatic machines } \\
\text { in the manufacturing } \\
\text { line }\end{array}$ & $\begin{array}{l}\text { accuracy }(95 \% \text { - Random Forest), } \\
\text { but the precision is low ( } 38 \%) \\
\text { which implies false alarms } \\
\text { recall ( } 74 \% \text { - Neural Networks) }\end{array}$ & fault prediction \\
\hline & & $\begin{array}{l}\text { Simulation } \\
+ \text { multi-sensor fusion }\end{array}$ & $\begin{array}{l}\text { Multi sensor } \\
\text { hard/soft data fusion }\end{array}$ & Aerospace & $\begin{array}{l}\text { Aerospace Industry } \\
\text { Manufacturing }\end{array}$ & $\begin{array}{l}\text { digital twin concept and many } \\
\text { levels of fusion for hard/soft data }\end{array}$ & $\begin{array}{l}\text { health status } \\
\text { estimation and } \\
\text { maintenance }\end{array}$ \\
\hline
\end{tabular}

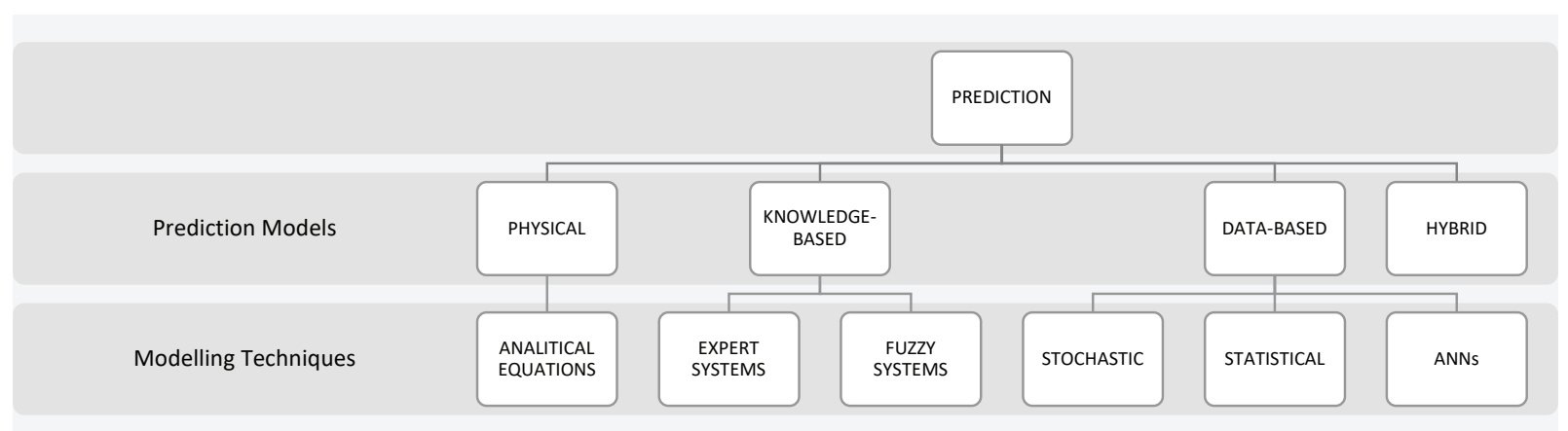

Fig. 1. Taxonomy of Prediction Models

conditions which is monitored continuously generates two types of data: event data and condition-monitoring data. Event data represent fault events which are considered critical to the system, and diagnostics messages when the events are alarm messages that described the item status. Events are triggered by the software component that monitors the item based on the item status information. Condition monitored data is collected every time when the events are triggered in order to form the context of the associated events and to ease their interpretation. Event data is characterized by attributes like type (i.e. fault, alarm, diagnostic), timestamp, item/subcomponent where the event was triggered, severity, duration, and textual description, among many other possible attributes. After the acquisition process, the data sets must be preprocessed as they exhibit uncertainties that may affect the learning model performance. Data preprocessing and feature extraction techniques are relevant in building reliable data driven models. Processing the raw data before modeling is improving the performance of the learned model. In practice, raw data in the form of sensor signals are complex and related information about the degradation process of the monitored component is not always available. Therefore, preprocessing raw sensor data is a mandatory step before building maintenance models. Generally, data processing methods can be divided into two main tasks, namely processing and data analysis. After processing the raw data coming from sensors, the resulting heterogeneous data may be categorized in the following types, depending on the quality of information they provide: (i) competitive, or redundant sensor data; (ii) cooperative, non-overlapping but partial sensor data; (iii) complementary, overlapping and partial sensor data; and (iv) independent, unrelated sensor data. Feature engineering is the next step prior to modeling the data. A feature is considered to be a predictive attribute for the model, such as temperature, pressure, vibration, etc. It is a good practice that the features extracted from the sensor data to comply with the following requirements: (i) features should 
contain information required to distinguish between potential faults; (ii) features should not take into account the irrelevant variability which might be mixed in the sensor signals; and (iii) features should be limited in number to allow efficient computation.

2) Model Development: ML techniques for predictive maintenance comprise two main approaches: (i) supervised learning, where the information about the occurrence of failures is present in the modeling data set; and (ii) unsupervised learning, where only the process information is available and no historic maintenance data exists. In an operational environment, predictive maintenance makes use of the following well-established techniques from ML field: (i) classification algorithms which are used to represent groups of normal and abnormal health status of the item under observation: Decision Tree, Random Forest, Nearest Neighbors, SVMs and HMMs; (ii) regression algorithms; and (iii) clustering methods with anomaly detection algorithms, as presented in Figure 2. Binary classification algorithms are used to predict the probability that a piece of equipment fails within a future time period. The business requirements, the analyzed available data and the domain expert make estimation for e.g. (i) minimum lead time required to replace components, deploy resources and perform maintenance actions in order to avoid a problem that is likely to occur in the future time period; or (ii) minimum count of events that can be triggered before a critical problem occurs. Multi-class classification algorithms are used for making predictions in the following possible scenarios: (i) defining a plan maintenance schedule i.e. estimation of the time intervals when an asset has the bigger probability to fail; (ii) monitoring the health status of an asset i.e. estimation of the probability that an asset will fail due to a specific cause /root problem; and (iii) prediction that an asset will fail due to a specific type of failure. In this case, a set of prescriptive maintenance actions can be considered for each of the previously identified set of failures. Another type of algorithms for classification are the multiple classifiers which can be used in the process of knowledge discovery to discern particular patterns of data degradation for an asset or for a process. The benefits of the multiple classifiers reside in allowing the planning of the maintenance schedules using a statistical cost minimization approach. Regression models are typically used to compute the RUL of an item, as presented in [8]. RUL is defined as the amount of time that an asset is operational before the next failure occurs. The operational historical data is needed because the RUL calculation is not possible without knowing how long the asset has survived before a failure. Autoregressive models such as ARMA models assume that all future values are linear functions of past observations. e.g. fault predictions. A data-based ANN approach is recommended to be used for information clustering when there is no knowledge or understanding about the monitored system e.g. [8] and [10].

3) Multimodal Machine Learning Methods: Multimodality is defined by [36] as referring to the way something happens, or is experienced: we read textual information, we see objects and we hear sounds, we feel textures and smell odors. All these perceptions represent modalities. A research problem, application or data set is multimodal when it includes multiple such modalities. In order to understand and to make sense of the world around us, A.I. techniques, in particular, multimodal machine learning (MML), must be able to interpret multimodal information and further to reason about it and make decisions. MML is a multi-disciplinary field of research which builds models that process and relate information from multiple modalities, as defined in [36]. The main idea is that data from different sensor sources provide different representations of the same phenomena. In MML literature, this is known as multimodal, multi-view, multi-representation or multi-source learning, as described in [37]. The main multimodal ML methods were identified and defined in [36] i.e. representation, translation, alignment, fusion, and co-learning. Their definitions according with [36] and [37] are listed in Table V. Understanding the capabilities and challenges of existing multimodal data fusion methods and techniques has the potential to deliver better data analysis tools across all domains, including the maintenance quality and management field of research. A relevant research challenge for the multimodal data fusion perspective is to identify patterns and commons governance rules that can be used to apply the appropriate multimodal data fusion technique for an application specific context or for a data set. Reference [38] arguments that data fusion is a multidisciplinary research area with ideas raised from many diverse research fields such as signal processing, information theory, statistical estimation and inference, and artificial intelligence. Data fusion appeared in the literature as mathematical models for data manipulation. The diversity of the research fields is indeed reflected in the reviews of maintenance techniques in Tables II, III and IV. Multimodal data fusion represents the integration of information from multiple modalities, with the goal of (i) making a prediction; and (ii) retrieving new insights from the joined knowledge, as defined by [36]. There are many approaches to data fusion, as the topic dates back in the 90es. The model-agnostic technique to data fusion is discussed in [36] and [39] and later, described by [37], which also lays the grounds for the multimodal data fusion formal theory. Multimodal data fusion has the direct economic impact in the implementation of maintenance techniques which are based on aggregation data from heterogeneous sources into actionable decisions for maintenance purposes. Multimodal data fusion represents the core concept in MML, as argued in [36] [39]. The model-agnostic data fusion types that are used in the operational environment are listed in Table VI. The reference [37] lays the grounds for the multimodal data fusion theory by giving a solution to the research problem of determining the appropriate type of data fusion for a specific application context or for a data set. On his view, the main challenge in multimodal data fusion research resolves around the dependency-problem i.e. the arguments for choosing a specific type of data fusion. The assumption is that the optimal fusion type to be employed in an operational environment depends on the level we expect to see a dependency between the inputs in the modalities: (i) feature-based fusion assumes a 


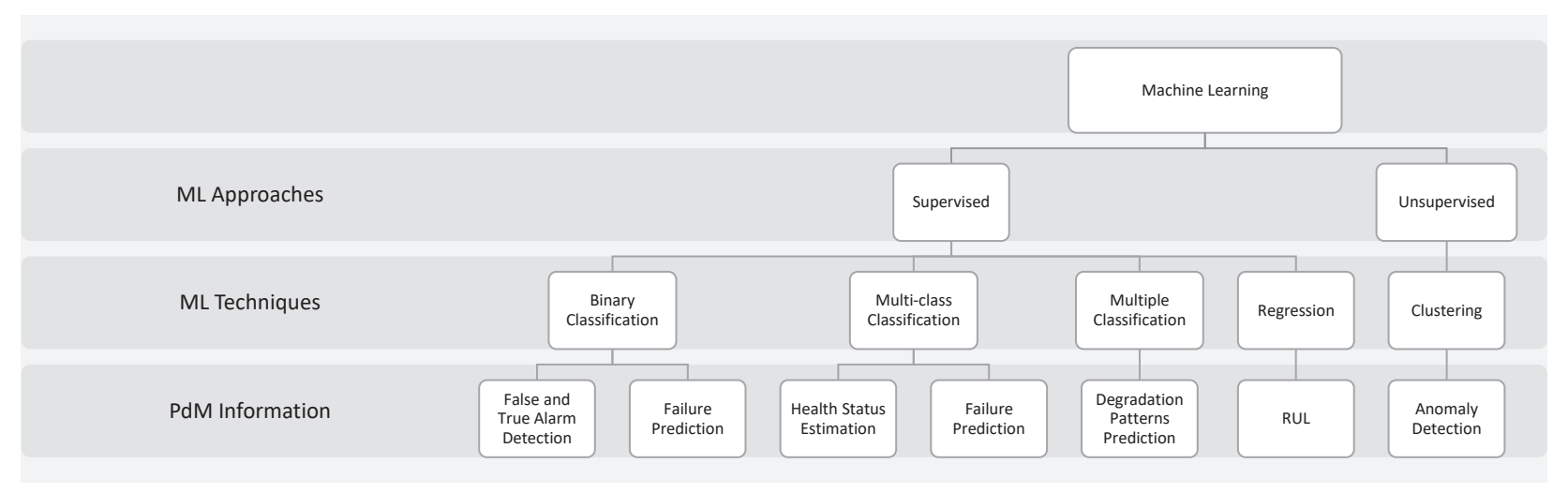

Fig. 2. Machine Learning Techniques for Predictive Maintenance

TABLE V

Multimodal Machine Learning (MML) Methods

\begin{tabular}{|c|c|}
\hline Representation & Learning to represent heterogeneous information in a unitary way, easy to be understood and processed by a learning model. \\
\hline Translation & Mapping the information from one modality to another in a most accurate way. \\
\hline Alignment & Identifying the inherent relations between sub-components. It also implies dealing with similarity measurements. \\
\hline Fusion & Joining/combining in a meaningful way the information from different modalities. \\
\hline Co-learning & Transferring knowledge among modalities: the modality with limited resources can benefit from another with more information. \\
\hline
\end{tabular}

dependency at the lowest level of features (or raw input unprocessed data), (ii) intermediate-fusion assumes a dependency at a more abstract, semantic level; and (iii) decision-based fusion assumes no dependency at all in the input, but only later at the level of decisions. The above described assumption has the following implications, as argued in [47]: (i) there are no established, standard methods to identify feature dependencies in multiple sensors and modalities; (ii) the technology exists, but there are no standard methods to extract unbiased feature from raw data, and therefore deep learning methods are preferred; (iii) there are basic techniques to handle modality fusion when dealing with missing information; (iv) it is unclear what are the relevant features to be learned, in the sense that a trial-and-error process of feature engineering is employed for the shallow ML algorithms, i.e. Decision Tree, SVM, kNN; and (v) multimodal data fusion best practices i.e. data sets, fusion algorithms, success stories, training and evaluation of results, should be recorded and shared. Moreover, the review of existing proposals for data fusion techniques and frameworks clearly shows that the actual trend for maintenance engineering is cloud maintenance i.e. maintenance-as-a-service, as argued in [2] and [11]. The envisioned platform is seen as a management system of smart services i.e. data-analysisas-a-service, prognostics-as-a-service or data-as-a-service, that represent better solutions in terms of technology, performance, and costs. The list of challenges continues with: (vi) the absence of a clearly defined generic framework for smart services that standardize the usage of a data fusion pipeline it is clear that in an operational environment more than one data fusion techniques should be applied; (vii) there are no standard techniques for dealing with temporal and spatial (context) data alignment and synchronization, i.e. the ontology-based proposal of [44] assumes some benefits due to knowledge access, reasoning and re-use of ontology web-standards; and (viii) lack of research studies to analyze the performance of ML algorithms in a cloud environment.

4) Model Evaluation: One challenge for evaluating the performance of learning models is represented by the availability of data: when the data set is not large enough to provide sufficient data quantities for the data training validation and test sets, then methods such as k-fold cross validation and bootstrapping are used to simulate new data. K-fold validation is used to split the original dataset into $\mathrm{k}$ folds, and run the learning algorithm $\mathrm{k}$ times. Another challenge on model evaluation is represented by the skew or imbalanced data. In any maintenance scenario, the minority data class is represented by the abnormal data i.e. the event-faults. Therefore, in the case of fault prediction, the algorithm needs to identify only a small group of data from the overall historical data. Incorrectly predicting a positive class as a negative may lead to a greater cost than the reverse situation, i.e. the problem of the asymmetric cost. Consequently, performance metrics based on the confusion table are used to evaluate how accurate the algorithm is. Boosted methods, such as boosted decision trees are also used as algorithms for solving the imbalanced data problem. Consequently, the time-dependent split of the imbalanced data is useful for avoiding data overfitting on classification models for fault predictions, and on regression models for predicting the RUL. Other problems to be avoided when evaluating a learning model are variance and bias. The 
TABLE VI

MODEL-AGNOSTIC FUSION TYPES

\begin{tabular}{|c|c|}
\hline Feature-based (Early Fusion) & Features from all the modalities are concatenated as one long input and trained by a single learner. \\
\hline Intermediate (Hybrid Fusion) & $\begin{array}{c}\text { There is a single learning model which is trained with a preprocessed input from modalities in the fused layer. } \\
\text { It is implemented by neural networks and multi-kernel support vector machines algorithms. }\end{array}$ \\
\hline $\begin{array}{c}\text { Decision-Based (Late Fusion) } \\
\text { Each modality is trained with a different learning model that independently makes a decision. All decisions } \\
\text { generated by learning models are later combined based on a fusing schema. }\end{array}$ & \begin{tabular}{c} 
Eached \\
\hline
\end{tabular}
\end{tabular}

reference [42] addresses these problems suggesting appropriate solutions.

\section{Discussions}

The present work reviews maintenance approaches with applicability in the industrial environment. The aim is to identify potential sources and ideas for delivering better data analysis tools and techniques for the optimization of the industrial maintenance processes. Past works on maintenance approaches show that maintenance actions are performed by employing various prediction models and modeling techniques. However, the existent literature does not inform us to which extent the new A.I. technology based on ML methods and techniques is influencing and changing the maintenance approaches in the industrial setup. Consequently, we provide an analytical literature review showing first that among all the existent approaches to maintenance, each of them varying in terms of efficiency and complexity, predictive maintenance seems to best fit the needs of a highly competitive industry setup. Next, we consider ML to be a prediction methodology and we show that ML methods enhance industrial maintenance with a critical component of intelligence: prediction. The approach we envision for the optimization of predictive maintenance actions investigates the MML perspective and consequently uses a variety of multimodal ML methods that study both live and historical information, in order to learn prognostics data and to make accurate diagnostics and predictions. Based on the surveyed literature we construct taxonomies that cover the main predictive models and their modeling techniques relative to maintenance goals. We show that among all the prediction models, the data driven, statistical inference based ML approaches are the most suitable to deal with big volumes of heterogeneous data. Their acceptance in the field is mainly due to the fact that prediction is easier than model inference i.e. the ML models are performing tests to check how well a learning model which is trained on a data set is able to predict new data. This allows ML algorithms to easily work with larger volumes of complex data. However, a critical analysis of ML algorithms and of the sensor data sets used for maintenance will directly show that there are no optimal ML models that always outperform all the other. Usually, their efficiency is based on the type of training data distribution. On its turn, multimodality is presented as an efficient ML method of combining data from multiple, diverse modalities and sources. Its main goals are: making better predictions and retrieving new insights from the combined knowledge. A model-agnostic taxonomy of the reviewed multimodal ML fusion methods is presented together with appropriate solu- tions for optimal usage. In particular, we distinguish among: (i) feature-based fusion or early fusion a basic concatenation of features belonging to different modalities; (ii) intermediatefusion typical for algorithms implemented by the artificial neuronal networks or by multi-kernel support vector machines; and (iii) decision-based fusion which applies a learning model for each modality independently, and the fusion takes place only at the decision level. Past works present multimodal fusion strategies made in an ad-hoc way, without following some standard implementation lines. We highlight the fact that there is a need for standardized solutions in applying multimodal ML methods for maintenance purposes. Moreover, we show that analyzing only the technical aspect i.e. the multimodal ML perspective, for improving the quality of maintenance is not sufficient. The connections with the economic aspect and the conformity of data science projects with industrial standards like CRISP-DM and Industrial Data Space are relevant. Consequently, we argue that quality of maintenance in an industrial setup can be improved only when in the development of a generalized architecture for maintenance purposes the following aspects are taken into consideration: (i) the technological aspect which recognizes the potential of multimodal ML methods for maintenance purposes; (ii) the business aspect which envisions a structured development of the implementation works starting with the business model's conceptualization, and assuring its conformity with the industry standards; and (iii) the economic aspect which follows the classical optimization concerns relative to maintenance costs. Future works are planned to analyze the usage of multimodal ML methods combined with semantic technologies in a cloud-oriented environment. The goal is to overcome the problem of sensor integration for efficient data analysis. We recognize that the actual trend for maintenance engineering is cloud maintenance. Within this context, the envisioned digital platform is seen as a management system of smart services i.e. prediction-as-a-service and maintenanceas-a-service, with expected benefits in terms of technology, performance and costs.

\section{REFERENCES}

[1] G. A. Susto, S. Mcloone, S. Pampuri, A. Benghi, and A. Schirru, "Machine Learning for Predictive Maintenance: A Multiple Classifier Approach", IEEE Transactions on Ind. Inf. 11(3), 2015, pp. 812-820, https://doi.org/10.1109/TII.2014.2349359.

[2] Z. Liu, M. Norbert, and M. Nezih, The role of Data Fusion in predictive maintenance using Digital Twin, in AIP Conference Proceedings 1949(1):02023, 2018, https://doi.org/10.1063/1.5031520.

[3] G. Manco, E. Ritacco, P. Rullo, L. Galluci, W. Astill, D. Kimber, and M. Antoneli,Fault detection and explanation through big data analysis on sensor streams, in Expert Syst. Appl. 87, 2017, pp. 141-156, https://doi.org/10.1016/j.eswa.2017.05.079. 
[4] G. Niu and H. Li, IETM centered intelligent maintenance system integrating fuzzy semantic inference and data fusion, in Microelectron. Reliab. 75, 2017, pp. 197-204, https://doi.org/10.1016/j.microrel.2017.03.015.

[5] T. Widmer, A. Klein, P. Wachter, and S. Meyl, Predicting Material Requirements in the Automotive Industry using Data Mining, in BIS, 2019, pp. 582-588

[6] Ł. Sobaszek, A. Gola, and E. Kozłowski, Application of survival function in robust scheduling of production jobs, in FedCSIS 2017, ACSIS Vol. 11, 2017, pp. 575-578, http://dx.doi.org/10.15439/2017F276.

[7] Ł. Sobaszek, A. Gola, and E. Kozłowski, Job-shop scheduling with machine breakdown prediction under completion time constraint, in FedCSIS 2018, ACSIS, Vol. 15, 2018, pp. 437-440, http://dx.doi.org/10.15439/2018F83.

[8] L. Guo, N. Li, F. Jia, Y. Lei, and J. Lin, A recurrent neural network based health indicator for remaining useful life prediction of bearings, in Neurocomputing 240, 2017, pp. 98-109, https://doi.org/ 10.1016/j.neucom.2017.02.045

[9] R. Acorsi, R. Manzini, P. Pascarella, M. Patella, and S. Sassi, Data Mining and Machine Learning for Condition-based Maintenance, in Int. Conf. on Flexible Automation and Intelligent Manufacturing 11, 2017, pp. 1153-1161, https://doi.org/10.1016/j.promfg.2017.07.239.

[10] M. Safizadeh and S. Latifi, Using multisensory data fusion for vibration fault diagnosis of rolling element bearings by accelerometer and load cell, in Inf. Fusion 18, 2014, pp. 1-8, https://doi.org/10.1016/j.inffus.2013.10.002.

[11] B. Schmidt, U. Sandberg and, L. Wang, Next generation condition based Predictive Maintenance, in Methods 13306, 2014, pp. 4-11.

[12] M. Schenk, Instandhaltung technicher Systeme, 2010.

[13] B. Otto, S. Auer, J. Cirullies, J. Jürjens, N. Menz, J. Schon, and S. Wenzel, Industrial Data Space Digital soveregnity over data, in Fraunhofer Gesellschaft zur Förderung der angewandten Forschung, 2016.

[14] DIN EN-13306. DIN Standards Publication Maintenance Begriffe der Instandhaltung/Maintenance terminology, 2010.

[15] DIN EN-31051. DIN Standards Publication Maintenance Grundlage der Instandhaltung/Fundamentals of Maintenance, 2012.

[16] A.R. Hevner, S.T. March, J. Park, and S. Ram, Design science in information system research, MIS Q. 28(1), 2004, pp. 75-105.

[17] B. J. Oates, Researching Information Systems and Computing, Sage Publications Ltd., 2006.

[18] K. Peffers, T. Tuunanen, M. Rothenberger and S. Chatterjee, A Design Science Research Methodology for Information Systems Research, in J. Manage. Inf. Syst. 24(3), 2007, pp. 45-77, https://doi.org/10.2753/MIS0742-1222240302.

[19] C. Bunks, D. McCarthy, and T. Al-Ani, Condition-based Maintenance of machines using hidden Markov Models, in NAMRC 32, 2004, pp. 597-612, https://doi.org/10.1006/mssp.2000.1309.

[20] P. Deuszkiewick and S. Radkowski, On-line condition monitoring of a power transmission unit of a rail vehicle, in Mechanical Systems and Signal Processing 17(6), 2003, pp. 1321-1334, https://doi.org/10.1006/mssp.2002.1578.

[21] Y. Hao, J. Sun, G. Yang and J. Bai, The Application of Support Vector Machines to Gas Turbines Performance Diagnosis, in Chinese Journal of Aeronautics 18 (1), 2005, pp. 15-19, https://doi.org/10.1016/S10009361(11)60276-8.

[22] P. Baraldi, E. Zio and F. di Maio, Unsupervised Clustering for Fault Diagnostics in Nuclear Power Plants Components, in Int. Journal of Comp. Intelligent Systems 6(4), 2014, pp. 764-777, https://doi.org/10.1080/18756891.2013.804145.

[23] A. Alexandru, Using Expert Systems for Fault Detection and Diagnosis in Industrial Applications, 1998.

[24] A. Krishnakumari, A. Elayaperumal, M. Saravanan, and C. Arvindan Fault diagnostics of spur gear using decision tree and fuzzy classifier, in Int. J. Adv. Manuf. Technol. 89 (9-12), 2017, pp. 3487-3494, https://doi.org/10.1007/s00170-016-9307-8.

[25] V.H. Jaramillo, J.R. Ottewill, R. Dudek, D. Lepiarczyk, and P. Pawlik, Condition monitoring of distributed systems using two-stage Bayesian inference data fusion, in Mech. Syst. Signal Process. 87, 2017, pp. 91110, https://doi.org/10.1016/j.ymssp.2016.10.004.

[26] C. Liu, Y. Li, G. Zhou, and W. Shen, A sensor fusion and support vector machine based approach for recognition of complex machining conditions, in Journal of Intelligent Manufacturing, 2016, pp. 1-14, https://doi.org/10.1007/s10845-016-1209-y.
[27] A. Diez, N.L.D. Khoa, M.M. Alamdari, Y. Wang, F. Chen, and P. Runcie, A clustering approach for structural health monitoring on bridges, in J. Civil Struct. Health Monitoring 6 (3), 2016, pp. 429-445.

[28] C. Li, R.-V. Sánchez, G. Zurita, M. Cerrada, and D. Cabrera, Fault diagnosis for rotating machinery using vibration measurement deep statistical feature learning, in Sensors 16 (6): 895, 2016, pp. 1-19.

[29] Q. (C.) Liu and H.P. (B.) Wang, A case study on multisensory data fusion for imbalanced diagnosis of rotating machinery, in AI EDAM 15(3), 2001, pp. 203-2010.

[30] A. Xenakis, A. Karageorgos, E. Lallas, A.E. Chis, and H. GonzalezVelez, Towards Distributed IoT/Cloud based Fault Detection and Maintenance in Industrial Automation, in EDI40, 2019, pp. 683-690, https://doi.org/10.1016/j.procs.2019.04.091.

[31] A. Mosallam, K. Medjaher, and N. Zerhouni, Data-driven prognostic method based on Bayesian approaches for direct remaining useful life prediction, in J. Intell. Manuf. 27 (5), 2016, pp. 1037-1048, https://doi.org/10.1007/s10845-014-0933-4.

[32] E. F. Alsina, M. Chica, K. Trawinski, and A. Regattieri, On the use of Machine Learning methods to predict component reliability from datadriven industrial case studies, in Int. J. Adv. Manufacturing Technology, (94), 2018, pp. 2419-2433, https://doi.org/10.1007/s00170-017-1039-X.

[33] L. Cristaldi, G. Leone, R. Ottoboni, S. Subbiah, and S. Tur rin, A comparative study on data-driven prognostic approaches using fleet knowledge, in IEEE International Conference on Instrumentation and Measurement Technology (I2MTC), 2016, pp. 1-6, https://doi.org/10.1109/I2MTC.2016.7520371.

[34] C. F. Baban, M. Baban, and M.D. Suteu, Using a fuzzy logic approach for the predictive maintenance of textile machines, in J. Intell. Fuzzy Syst. 30 (2), 2016, pp. 999-1006, https://doi.org/10.3233/IFS-151822

[35] W. Cui, Z. Lu, C. Li, and X. Han, A proactive approach to solve integrated production scheduling and maintenance planning problem in flow shops, in Comput. Ind. Eng. 115, 2018, pp. 342-353, https://doi.org/10.1016/j.cie.2017.11.020.

[36] T. Baltrusaitis, C. Ahuja, and L. Morency, Multimodal Machine Learning: A Survey and Taxonomy, in IEEE transactions on pattern analysis and machine intelligence, 2017, pp. 423-443, https://doi.org/10.1109/TPAMI.2018.2798607.

[37] E. Alpaydin, Classifying multimodal data" in The Handbook of Multimodal-Multisensor Interfaces, Ed. Sharon Oviatt, Björn Schuller, Philip R. Cohen, Daniel Sonntag, Geranimos Potamianos, and Antonio Krüger, in Association for Computing Machinery and Morgan \& Claypool, NY, 2018, pp. 49-69, https://10.1145/3107990.3107994.

[38] B. Khaleghi, F. Karray, A. Khamis, and S. N. Razavi, Multisensor Data Fusion: A review of the State-of-the-Art, in Information Fusion 14, 2013, pp. 28-44, https://doi.org/10.1016/j.inffus.2011.08.001.

[39] Y. Bengio, A. Courville, and P. Vincent, Representation learning: a review and new perspectives, Technical report. U Montreal, 35(8), pp. 1798-1828, 2013, https://doi.org/10.1109/TPAMI.2013.50.

[40] N. Srivastava and R. Salakhutdinov, Multimodal learning with Multimodal Boltzmann Machines, in Advances in Neural Information Processing Systems, 2012, pp. 2222-2230.

[41] A. Zheng, Evaluating machine Learning Models A Beginners Guide to Key Concepts and Pitfalls. OReilly Media, 2015, ISBN 978-1-49193246-9.

[42] A. Ng, Machine Learning, Online Course offered by Stanford University, 2010.

[43] B. Schmidt, W. Lihui, and D. Galar, Semantic Framework for PdM in a cloud environment, in CIRP ICME 62, 2017, pp. 582-588, https://doi.org/10.1016/j.procir.2016.06.047

[44] H. Seidgar, M. Zandieh, and I. Mahdavi, An efficient metaheuristic algorithm for scheduling a two-stage assembly flow shop problem with preventive maintenance activities and reliability approach, in Int. J. Ind. Syst. Eng. 26(1), 2017, pp. 16-41, https://doi.org/10.1504/IJISE.2017.083180.

[45] A. Diez-Olivan, J. del Ser, D. Galar, and B. Sierra, Data fusion and machine learning for industrial prognosis: Trends and perspectives towards Industry 4.0, in Int. J. Inf. Fusion 50, 2019, pp. 92-111, https://doi.org/10.1016/j.inffus.2018.10.005

[46] P.H. Foo and G.W. Ng, High-level Information Fusion: An Overview, in Journal of Advances in Information Fusion, 8(1), 2013, pp. 33-72, https://doi.org/10.1.1.360.6651.

[47] C.-A. Chou, X. Jin, A. Müller, and S. Ostadabbas, (MMDF) Multimoda Data Fusion Workshop Report, 2018. 